\title{
Conhecimentos dos Médicos Residentes de Ginecologia e Obstetrícia sobre Contracepção Hormonal em Situações Especiais
}

\section{Knowledge on Hormonal Contraception in Special Situations Among Gynecology and Obstetrics Medical Residents}

\author{
PALAVRAS-CHAVE \\ - Anticoncepção. \\ - Ginecologia. \\ - Educação Médica. \\ - Organização Mundial de Saúde.
}

Recebido em: 02/06/2016

Aprovado em: 19/09/2016
Margareth Rocha Peixoto Giglio Gisele Pimenta Melo ${ }^{I}$ Vanessa Guerra Ferreira ${ }^{I}$ Marco Aurélio Albernaz Marília Oliveira Ribeiro ${ }^{I}$

\begin{abstract}
RESUMO
Introdução: Os anticoncepcionais hormonais $(A C H)$ vêm sendo utilizados e difundidos desde a década de 1960. Sua importância atualmente é inegável, pois eles são responsáveis pelo tratamento e prevenção de várias doenças ginecológicas. A evolução dos ACH consistiu na implantação de uma ampla variedade de contraceptivos, de diferentes dosagens, combinações e formas de administração. Com o intuito de orientar sobre a segurança dos vários métodos contraceptivos em contextos específicos, a Organização Mundial da Saúde (OMS) lançou o guia Critérios de Elegibilidade para o Uso de Contraceptivos. Objetivo: Este estudo objetiva avaliar o conhecimento dos residentes de Ginecologia e Obstetrícia de Goiânia a respeito da contracepção hormonal, de acordo com o guia Critérios de Elegibilidade para o Uso de Contraceptivos da OMS. Métodos: Foi realizado um estudo transversal, descritivo, com residentes de Ginecologia e Obstetrícia de dois hospitais de Goiânia (GO), um filantrópico e outro da Secretaria Estadual de Saúde de Goiás. Foi aplicado um questionário com perguntas de cunho epidemiológico e perguntas técnicas sobre indicações e contraindicações de determinados métodos contraceptivos nas seguintes situações especiais: cefaleias, hipertensão arterial sistêmica, tabagismo, tromboembolismo, trombose arterial e embolia pulmonar. Foi realizada análise univariada, e os dados foram dispostos em tabelas de frequência. Resultados: Os questionários foram respondidos por 33 residentes, com média de idade de 29,2 anos, dos quais 61\% afirmaram ter formação adequada sobre contracepção; $63 \%$ se sentiam aptos a prescrevê-los; e os residentes dos dois últimos anos se sentiram mais seguros $(82,4 \%)$ do que o grupo de recém-chegados à residência e os residentes do primeiro ano (75\%), porém não foi obtida diferença significativa entre eles. Conclusão: Os anos de residência médica não foram suficientes para melhorar significativamente os conhecimentos a respeito dos critérios de elegibilidade da OMS entre os grupos iniciais e finais do curso de residência, apesar de oferecerem maior segurança na prescrição.
\end{abstract}




\author{
KEYWORDS \\ - Contraception. \\ - Gynecology. \\ - Medical Education. \\ - World Health Organization.
}

\begin{abstract}
Introduction: Hormonal contraceptives have been available for use since the 1960s, with their importance now undeniable, thanks to their treatment and prevention of various gynecological diseases. The development of hormonal contraceptives has involved the implementation of a wide range of contraceptives, different dosages, combinations and forms of administration. In order to advise on the safety of the prescription of these drugs in special situations, the WHO launched a guide known as the "WHO Eligibility Criteria for the use of hormonal contraceptives". Objective: This study aims to evaluate knowledge among gynecology and obstetrics residents according to the WHO guidelines. Methods: A cross-sectional survey was administered to gynecology and obstetrics medical residents at two hospitals in Goiânia, with one hospital philanthropic and the other pertaining to the Goiás State Department of Health. A questionnaire was administered featuring epidemiological and technical questions on the indications and contraindications associated with the use of certain contraceptive methods in special conditions, such as headache, hypertension, smoking, thromboembolism, arterial thrombosis, and pulmonary embolism. A univariate analysis was performed, with the data organized into frequency tables. Results: The questionnaires were completed by 33 residents with a mean age of 29.2 , with $61 \%$ revealed to have received adequate training on contraception and $63 \%$ feeling able to prescribe hormonal contraceptive methods. Residents in the final two years felt safer (82.4\%) than the newcomer resident group and those in the first year (75\%), however a significant difference was not recorded. Conclusion: The number of years students had been resident was not enough to significantly improve knowledge on the WHO Eligibility Criteria, in our comparison between residents in the initial and final stages of the course, despite tallying safer prescription.
\end{abstract}

\section{INTRODUÇÃO}

A contracepção não é um conceito da era moderna. Sua história é milenar e foi praticada inicialmente por métodos contraceptivos rudimentares com o intuito de controlar não só a natalidade, mas também o infanticídio dos países há mais de dois mil anos ${ }^{1,2}$ - uma temática que já gerou desmedidas desavenças por questões religiosas, culturais, políticas e individuais ${ }^{3}$. Nesta perspectiva, resgatar a história dos métodos contraceptivos é reviver também um pouco a história da mulher e da liberdade sexual conquistada ao longo do tempo ${ }^{4-13}$.

$\mathrm{O}$ uso de anticoncepcionais hormonais $(\mathrm{ACH})$ teve seu marco na década de 1960, quando foi legalizado o primeiro contraceptivo hormonal nos Estados Unidos, correspondente a uma pílula combinada contendo $10 \mathrm{mcg}$ de progesterona (noretinodrel) e $150 \mathrm{mcg}$ de estrogênio (mestranol), que cerca de três anos após foi retirada do mercado por estar associada a alta incidência de tromboembolismo ${ }^{14,15}$.

Em vista disso, um estudo realizado na Inglaterra, que associou a ocorrência de tromboembolismo a alta dosagem do estrogênio, orientou que a dose de etinilestratiol (EE) dos contraceptivos fosse menor que $50 \mathrm{mcg}^{16,17}$. Desde então, na corrida pela diminuição do tromboembolismo, os contraceptivos foram diminuindo a dose do EE gradativamente, até chegar aos contraceptivos só de progestágenos e, mais recentemente, associando o $\beta$-estradiol (BE) a progestinas nos contraceptivos combinados mais modernos ${ }^{18,19}$.
Grandes mudanças também se fizeram no tocante às progestinas usadas isoladamente ou em associações com os estrogênios, EE e BE. A corrida aqui foi para encontrar um progestágeno com efeitos antimineralcorticoides e menores efeitos androgênicos ${ }^{20,21}$. Nesse sentido, podem-se dividir os progestágenos estruturalmente relacionados a três compostos: 19-nortestosterona, 17-hidroxiprogesterona e 17- $\alpha$-espironolactona. Os componentes estruturalmente relacionados à nortestosterona foram divididos em gerações, de acordo com a diminuição da afinidade pelo receptor testosterônico e efeito androgênico. Na primeira geração temos: noretinodel; noretindrona/noretisterona; linestrenol; norgestrienona. Na segunda: norgestrel; levonorgestrel; e na terceira: desogestrel, gestodeno e norgestimato. Com relação à 17-hidroxiprogesterona, encontra-se um derivado progestagênico direto, contando-se com os seguintes fármacos: acetato de medroxiprogesterona; mesogestrol; acetato de clormadinoma; e acetato de ciproterona. Entre esses compostos, o acetato de ciproterona apresenta a maior ação antiandrogênica, e os derivados da primeira geração, os mais androgênicos, com os demais compostos intercalados entre esses dois ${ }^{22,23,24}$. O derivado da 17- $\alpha$-espironolactona, drospirenona, apresenta efeito antimineralocorticoide. Vale ressaltar também que os progestagênios, quando administrados isoladamente, afetam de forma mínima o sistema de coagulação, fazendo com que essa seja uma opção mais segura nas pacientes com as condições especiais citadas neste estudo ${ }^{15,25}$. 
Outro marco na história dos ACH ocorreu em 1995, quando foram divulgados os primeiros estudos segundo os quais contraceptivos orais combinados contendo progestágenos de terceira geração (gestodeno, desogestrel) se associavam a um risco duas vezes maior de trombose do que os que continham progestagênios de segunda geração (levonorgestrel) ${ }^{26,16}$. A partir de então, vários estudos têm demonstrado que, quanto mais antiandrogênico for um progestágeno, maior será a sinergia com o estrógeno em relação à ocorrência do tromboembolismo ${ }^{27}$.

Assim, neste processo de evolução, nas últimas décadas, os contraceptivos hormonais passaram por uma importante melhoria farmacológica, com a descoberta de preparados com novos compostos químicos e o lançamento de novas vias de utilização. Atualmente, é possível encontrar produtos contraceptivos hormonais (combinados e isolados) sob a forma de comprimidos, injeções, implantes subcutâneos, adesivos transdérmicos, dispositivos intrauterinos (DIU) e os anéis vaginais que liberam hormônios $28,29,30$. Isso tudo tem permitido uma contracepção bem mais abrangente, plena e acessível, com melhoria dos serviços de planejamento familiar e maior liberdade para a prática sexual.

Além disso, é inegável a importância dos $\mathrm{ACH}$ no tratamento e prevenção de várias doenças ginecológicas, como dismenorreia, anemia ferropriva, tensão pré-menstrual, menorragia, gravidez ectópica, cistos ovarianos, doenças benignas das mamas, câncer de endométrio e câncer de ovário, além do controle da síndrome dos ovários policísticos, endometriose e a redução do número de abortos provocados ${ }^{31}$.

Com o intuito de orientar sobre a segurança dos vários métodos contraceptivos em contextos específicos, a Organização Mundial da Saúde (OMS) lançou a primeira edição dos Critérios Médicos de Elegibilidade para Uso de Métodos Anticoncepcionais (CMEUA) em 1996, classificando-os em quatro categorias: na Categoria 1, o método pode ser utilizado sem qualquer restrição; na Categoria 2, o uso do método pode apresentar algum risco, habitualmente menor do que os benefícios decorrentes de seu uso, e, assim, pode ser empregado com cautela e precauções maiores, com acompanhamento clínico mais rigoroso; a Categoria 3 corresponde aos métodos que podem estar associados a um risco que supera os benefícios decorrentes de seu uso, podendo ser usado, contudo, se não houver outra opção disponível ou em situações em que a pessoa não aceita qualquer outra alternativa, mas desde que seja alertada sobre este fato, submetendo-se a uma vigilância médica muito rigorosa; a Categoria 4 constitui contraindicação absoluta ao método, pois determina um risco inaceitável à saúde ${ }^{28,32}$. Estes critérios têm sido atualizados à medida que novos conhecimentos vão sendo introduzidos pela medicina baseada em evidências.
Entretanto, apesar de todos esses avanços e dos inúmeros benefícios que vêm ocorrendo há mais de 50 anos na contracepção hormonal, a medicina baseada em evidências, aliada à prática clínica, aponta o fato de que muitas mulheres no mundo todo ainda receiam o uso de métodos contraceptivos hormonais por preconceito ou desconhecimento do tema. Da mesma forma, profissionais de saúde também deixam de prescrever ou orientar a contracepção hormonal pelos mesmos motivos, como evidenciado no estudo de Paz et al. ${ }^{5,33}$.

Outro estudo, realizado em Santa Catarina em 2008, que avaliou o conhecimento e consumo entre mulheres universitárias sobre a contracepção de emergência, mostrou que apenas $2,9 \%$ das mulheres adquiriram o medicamento mediante prescrição médica e que $35,3 \%$ receberam orientações no momento da compra, mostrando a importância não só do médico, mas de vários outros profissionais de saúde para a o consumo dos contraceptivos hormonais pela população em geral ${ }^{34}$.

Considerando-se a importância do tema, o presente estudo teve como objetivo avaliar o conhecimento dos médicos residentes de Ginecologia e Obstetrícia de Goiânia a respeito da contracepção hormonal envolvendo os CMEUA da OMS em situações específicas de cefaleias, tromboembolismo, embolia pulmonar, trombose arterial, hipertensão arterial sistêmica e tabagismo, bem como comparar e analisar os seus conhecimentos de acordo com o ano em que cursam a residência médica.

\section{METODOLOGIA}

Para atingir os objetivos propostos, foi realizado um estudo transversal, de prevalência, descritivo, no qual foram incluídos todos os médicos residentes de Ginecologia e Obstetrícia (GO) de dois hospitais de Goiânia, um filantrópico (HF) e a maternidade da Secretaria Estadual de Saúde de Goiás (MSES), responsáveis por cerca de $70 \%$ dos residentes de GO nesse município, no ano de 2016.

Este estudo faz parte de uma linha de pesquisa que vem avaliando os conhecimentos de profissionais da área médica, uma vez que já foi realizado um estudo entre alunos do internato de Medicina de uma Universidade de Goiás, publicado em $2015^{35}$

Três residentes $(8,4 \%)$ não foram incluídos no estudo porque se encontravam de férias ou ainda não haviam ingressado na residência no período da coleta dos dados. Todos os participantes assinaram o Termo de Consentimento Livre e Esclarecido para participação no estudo.

O instrumento de coleta de dados foi constituído por um questionário com perguntas abertas e fechadas, de cunho epidemiológico e referentes a segurança/insegurança na con- 
tracepção e motivos atribuídos a isso (total de 17 perguntas). Além disso, havia nove perguntas técnicas fechadas a respeito da contracepção nas seguintes situações de risco: cefaleias, hipertensão arterial sistêmica, tabagismo, tromboembolismo, trombose arterial e embolia pulmonar, como teste de múltipla escolha. Essas perguntas foram baseadas nos CMEUA da OMS.

Cada questão sobre situações técnicas relativas à prescrição dos ACH era de múltipla escolha e contava com o mesmo grupo de opções para serem assinaladas: (A) anticoncepcional oral combinado; (B) anticoncepcional oral com progestágeno isolado; (C) DIU de levonorgestrel (mirena); (D) injetável mensal; (E) injetável trimestral; (F) anticoncepção hormonal combinada transdérmica (adesivo); $(G)$ anticoncepção hormonal combinada via vaginal; $(\mathrm{H})$ nenhuma das opções anteriores.

O médico residente deveria ser capaz de fazer a leitura do caso e prescrever corretamente todos os métodos permitidos à situação ou fazê-lo de forma parcial, identificando as contraindicações relativas e absolutas para cada situação. Foram consideradas corretas as assertivas B, C, E nas questões 20, 21, $22,25,27,28$, e as assertivas A, B, C, D, E, F e G nas questões 23,24 e 26.

Os questionários foram aplicados por duas alunas do $12^{\mathrm{O}}$ período do curso de Medicina da PUC-Goiás nos meses de fevereiro a março de 2016 no momento em que os residentes se encontravam reunidos para atividades teóricas da residência médica. Contudo, cerca de $35 \%$ dos médicos residentes tiveram que ser abordados isoladamente porque não estavam presentes nessas atividades. Durante a aplicação dos questionários não foram permitidas consultas ou perguntas sobre o assunto em questão.

Um banco de dados foi construído e analisado com o software SPSS 19.0. Os resultados das análises univariadas são apresentados em tabelas de frequência. Como o grupo de médicos residentes estudados era pequeno, para a análise de alguns aspectos eles foram divididos em dois grupos: um formado pelos médicos residentes recém-chegados e pelos do primeiro ano, e o outro formado pelos médicos residentes dos dois últimos anos. Os médicos residentes recém-chegados correspondem àqueles que recentemente entraram para o serviço de residência médica, já que o período de estudo foi realizado na transição do início da residência médica, nos períodos de fevereiro a março.

O presente estudo atendeu às determinações da Resolução 466/12 da Comissão Nacional de Ética em Pesquisa em Seres Humanos, tendo sido analisado e aprovado pelo Comitê de Ética da PUC-Goiás, com o Protocolo nº 51780415.1.0000.0037.

\section{RESULTADOS}

Foram estudados 33 médicos residentes $(91,6 \%)$, sendo que 13 (39\%) eram do serviço de residência do HF e 20 (61\%) da MSES. Todos os médicos residentes de GO estudados residiam na grande Goiânia; a maioria era de mulheres $(81,8 \%)$, com média de idade de 29,2 anos (24 a 42 anos). Sete (21,2\%) alunos estavam ingressando no primeiro ano de residência, 9 $(27,3 \%)$ terminando o primeiro ano, 10 (30,3\%) o segundo ano, e $7(20,9 \%)$ o terceiro ano. A Tabela 1 mostra os demais dados referentes às características epidemiológicas dos estudados.

\begin{tabular}{|c|c|c|}
\hline \multicolumn{3}{|c|}{$\begin{array}{c}\text { Características epidemiológicas dos médicos residentes } \\
\text { em Ginecologia e Obstetrícia, Goiânia - GO, } 2016\end{array}$} \\
\hline \multicolumn{3}{|l|}{ 1. Hospitais } \\
\hline Hospital filantrópico & 13 & 39,4 \\
\hline Maternidade da Secretaria Estadual de Saúde & 20 & 60,6 \\
\hline \multicolumn{3}{|l|}{ 2. Sexo } \\
\hline Feminino & 27 & 81,8 \\
\hline Masculino & 6 & 18,2 \\
\hline \multicolumn{3}{|l|}{ 3. Idade (anos) } \\
\hline$<25$ & 1 & 3 \\
\hline $25-28$ & 21 & 48,5 \\
\hline$>28$ & 11 & 33,3 \\
\hline \multicolumn{3}{|l|}{ 4. Estado civil } \\
\hline Casado & 11 & 33,3 \\
\hline Solteiro & 22 & 66,7 \\
\hline \multicolumn{3}{|l|}{ 5. Raça } \\
\hline Branca & 24 & 72,7 \\
\hline Parda & 8 & 24,2 \\
\hline Preta & 1 & 3 \\
\hline
\end{tabular}

Cerca de 51,5\% dos médicos entraram nos serviços de residência médica imediatamente após o término do curso de Medicina e 30\% ingressaram entre seis meses a um ano após o término da graduação. Observou-se que 84,8\% frequentaram algum preparatório para residência médica.

Oitenta e sete por cento dos médicos residentes avaliados usavam algum método contraceptivo, sendo que o mais utilizado, correspondendo a 75,7\%, foi o contraceptivo hormonal oral.

Quando questionados se tiveram formação adequada quanto à contracepção, cerca de $61 \%$ disseram que sim e 39\% que não. Sessenta e três por cento se sentiam aptos a prescrever contraceptivos hormonais e, apesar de os médicos residentes dos dois últimos anos se sentirem mais seguros $(82,4 \%)$ do que o grupo de recém-chegados na residência e os médicos residentes do primeiro ano (75\%), não houve diferença estatística entre os grupos. 
O principal motivo pelo qual os médicos não se sentiam capacitados para prescrever os contraceptivos hormonais foi a falta de conhecimento e/ou prática, tanto no grupo de médicos residentes recém-chegados e do primeiro ano, como no grupo dos dois últimos anos de residência, não havendo diferenças estatísticas entre eles.

Dos $7(21,2 \%)$ médicos que referiram não ter tido discussão a respeito dos CMEUA da OMS durante a graduação, cerca de 33\% eram recém-chegados à residência médica. Dos 7 médi$\cos (21,2 \%)$ que referiram não ter tido discussão a respeito desses critérios durante a residência, $2(29 \%)$ eram do último ano. Quando questionados, os diretores do HF e da MSES informaram, respectivamente, que suas unidades não tinham serviço organizado de planejamento familiar e que, apesar de haver um serviço de planejamento na maternidade da SES/GO, esse era mais direcionado aos casos de esterilização cirúrgica.

\section{TABELA 2}

Frequência de acertos dos médicos residentes de Ginecologia e Obstetrícia, na prescrição de contraceptivos hormonais em situações especiais, quando foi considerado cada método, isoladamente, para cada questão, Goiânia - GO, 2016

\begin{tabular}{|c|c|c|c|c|}
\hline \multirow{2}{*}{ Situações } & \multicolumn{2}{|c|}{ Correto } & \multicolumn{2}{|c|}{ Errado } \\
\hline & $\mathbf{N}$ & $\%$ & $\mathbf{N}$ & $\%$ \\
\hline $\begin{array}{l}\text { 1. Que método(s) contraceptivo(s) você } \\
\text { indicaria para uma mulher na menacme com } \\
\text { hipertensão arterial sistêmica grave? }\end{array}$ & 28 & 84,8 & 5 & 15,2 \\
\hline $\begin{array}{l}\text { 2. Que método(s) contraceptivo(s) você } \\
\text { indicaria para uma mulher hipertensa leve e } \\
\text { controlada? }\end{array}$ & 26 & 78,8 & 7 & 21,2 \\
\hline $\begin{array}{l}\text { 3. Que método(s) contraceptivo(s) você } \\
\text { indicaria para uma paciente na menacme com } \\
\text { antecedente pessoal de trombose arterial? }\end{array}$ & 22 & 66,7 & 11 & 33,3 \\
\hline $\begin{array}{l}\text { 4. Que método(s) contraceptivo(s) } \\
\text { você indicaria para uma paciente com } \\
\text { antecedentes familiares de primeiro grau de } \\
\text { tromboembolismo arterial? }\end{array}$ & 29 & 87,9 & 4 & 12,1 \\
\hline $\begin{array}{l}\text { 5. Que método(s) contraceptivo(s) você } \\
\text { indicaria para uma paciente com antecedentes } \\
\text { familiares de primeiro grau de trombose venosa } \\
\text { profunda? }\end{array}$ & 30 & 90,9 & 3 & 9,1 \\
\hline $\begin{array}{l}\text { 6. Que método(s) contraceptivo(s) você } \\
\text { indicaria para uma paciente com antecedente } \\
\text { de trombose arterial ou tromboembolismo } \\
\text { pulmonar? }\end{array}$ & 19 & 57,6 & 14 & 42,4 \\
\hline $\begin{array}{l}\text { 7. Que método(s) contraceptivo(s) você } \\
\text { indicaria para uma paciente com cefaleia } \\
\text { moderada sem relação com menstruação e sem } \\
\text { sinais focais? }\end{array}$ & 32 & 97 & 1 & 3 \\
\hline $\begin{array}{l}\text { 8. Que método(s) contraceptivo(s) você } \\
\text { indicaria para uma paciente com cefaleia } \\
\text { moderada, acompanhada de escotomas e } \\
\text { parestesia de membro superior direito? }\end{array}$ & 27 & 81,8 & 6 & 18,2 \\
\hline $\begin{array}{l}\text { 9. Que método(s) contraceptivo(s) você } \\
\text { indicaria para uma paciente com mais de } 35 \\
\text { anos e tabagista ( }>15 \text { cigarros } / \text { dia)? }\end{array}$ & 26 & 78,8 & 7 & 21,2 \\
\hline
\end{tabular}

As Tabelas 2 e 3 mostram, por meio da frequência de acertos e erros, a avaliação do conhecimento dos médicos residentes de GO sobre a contracepção hormonal em situações especiais, de acordo com os CMEUA da OMS, analisando cada método de forma isolada e em conjunto, respectivamente. Não houve diferenças nas proporções de acertos, nas duas formas de análises, em relação aos anos de curso da residência médica.

\section{TABELA 3}

Frequência de acertos dos médicos residentes de Ginecologia e Obstetrícia na prescrição de contraceptivos hormonais em situações especiais, quando foram considerados todos os métodos corretos para cada questão, Goiânia - GO, 2016

\begin{tabular}{|c|c|c|c|c|}
\hline \multirow{2}{*}{ Situações } & \multicolumn{2}{|c|}{ Correto } & \multicolumn{2}{|c|}{ Errado } \\
\hline & $\mathbf{N}$ & $\%$ & $\mathbf{N}$ & $\%$ \\
\hline $\begin{array}{l}\text { 1. Que método(s) contraceptivo(s) você indicaria } \\
\text { para uma mulher na menacme com hipertensão } \\
\text { arterial sistêmica grave? }\end{array}$ & 8 & 24,2 & 25 & 75,8 \\
\hline $\begin{array}{l}\text { 2. Que método(s) contraceptivo(s) você indicaria } \\
\text { para uma mulher hipertensa leve e controlada? }\end{array}$ & 5 & 15,2 & 28 & 84,8 \\
\hline $\begin{array}{l}\text { 3. Que método(s) contraceptivo(s) você } \\
\text { indicaria para uma paciente na menacme com } \\
\text { antecedente pessoal de trombose arterial? }\end{array}$ & 6 & 18,2 & 27 & 81,8 \\
\hline $\begin{array}{l}\text { 4. Que método(s) contraceptivo(s) você indicaria } \\
\text { para uma paciente com antecedentes familiares } \\
\text { de primeiro grau de tromboembolismo arterial? }\end{array}$ & 5 & 15,2 & 28 & 84,8 \\
\hline $\begin{array}{l}\text { 5. Que método(s) contraceptivo(s) você } \\
\text { indicaria para uma paciente com antecedentes } \\
\text { familiares de primeiro grau de trombose venosa } \\
\text { profunda? }\end{array}$ & 6 & 18,2 & 27 & 81,8 \\
\hline $\begin{array}{l}\text { 6. Que método(s) contraceptivo(s) você indicaria } \\
\text { para uma paciente com antecedente pessoal } \\
\text { de trombose arterial ou tromboembolismo } \\
\text { pulmonar? }\end{array}$ & 4 & 12,1 & 29 & 87,9 \\
\hline $\begin{array}{l}\text { 7. Que método(s) contraceptivo(s) você indicaria } \\
\text { para uma paciente com cefaleia moderada sem } \\
\text { relação com menstruação e sem sinais focais? }\end{array}$ & 8 & 24,2 & 25 & 75,8 \\
\hline $\begin{array}{l}\text { 8. Que método(s) contraceptivo(s) você indicaria } \\
\text { para uma paciente com cefaleia moderada, } \\
\text { acompanhada de escotomas e parestesia de } \\
\text { membro superior direito? }\end{array}$ & 9 & 27,3 & 24 & 72,7 \\
\hline $\begin{array}{l}\text { 9. Que método(s) contraceptivo(s) você } \\
\text { indicaria para uma paciente com mais de } 35 \\
\text { anos e tabagista (>15 cigarros/dia)? }\end{array}$ & 7 & 21,2 & 26 & 78,8 \\
\hline
\end{tabular}

\section{DISCUSSÃO}

A falta de um consistente e preciso conhecimento sobre contracepção entre os profissionais de saúde tem o potencial de afetar drasticamente a assistência e o cuidado relacionados à contracepção, gerando importante impacto sobre a capacidade de prevenção de gravidezes não desejadas, como evidenciado no estudo de Dehlendorf et al. ${ }^{36}$. 
O conhecimento sobre os Critérios Médicos de Elegibilidade para o Uso de Anticoncepcionais da OMS é essencial para a prescrição dos ACH, evitando-se equívocos principalmente em se tratando de mulheres com condições especiais, como as avaliadas no presente estudo ${ }^{37}$.

Por outro lado, muitas mulheres com condições de saúde específicas são susceptíveis a terem opções contraceptivas hormonais coagidas ou interceptadas por falta de conhecimentos dos profissionais de saúde, visto que um ou outro método poderia ser consultado naquela circunstância, como mostrado nos estudos de Dehlendfort et al. ${ }^{36}$ e de Isaacs e Creinin ${ }^{38}$. Esse último estudo evidenciou que $14 \%$ das mulheres participantes haviam recebido informações inadequadas sobre contracepção, resultando na utilização de um método menos eficaz ou no uso incorreto do método contraceptivo ${ }^{38}$.

A maior contribuição do presente estudo foi mostrar que os anos de residência médica, embora ofereçam maior segurança aos médicos estudados em relação à prescrição de contraceptivos hormonais nas situações especiais, não foram suficientes para melhorar significativamente os conhecimentos a respeito dos CMEUA da OMS entre os grupos iniciais e finais do curso de residência, como mostram os resultados encontrados. Esses aspectos, ainda que possam ter sofrido influência do pequeno número de médicos residentes (o que prejudicou a avaliação entre os grupos categorizados por ano de residência), apontam a falta de momentos de aplicação prática dos conhecimentos.

Contudo, sob outra perspectiva, um estudo de Serrano et al., em 2009, que avaliaram cem residentes de diferentes anos de especialização em Ginecologia e Obstetrícia, evidenciou que os residentes do último ano, quando comparados aos do primeiro e segundo ano de residência, por apresentarem maior contato e prática nas prescrições de contraceptivos, desenvolveram maior visão e experiência na sua prática clínica ${ }^{33}$.

A residência médica em Ginecologia e Obstetrícia, por ser uma modalidade de ensino caracterizada por treinamento em serviço sob supervisão, em tempo integral, constitui um ótimo momento para o aprendizado e aperfeiçoamento dos conhecimentos na área da saúde reprodutiva ${ }^{39}$. Contudo, nem sempre os serviços de residência oferecem momentos específicos para a prática e sedimentação dos conhecimentos aprendidos nas discussões teóricas.

Os resultados encontrados no presente estudo parecem apontar esses aspectos, visto que a maioria dos médicos que se disse não preparada para a prescrição dos $\mathrm{ACH}$ informou como motivo para essa insegurança a falta de atividades práticas. De fato, as duas instituições estudadas não apresentavam serviços efetivos de planejamento familiar que poderiam for- necer o melhor momento para aplicação prática dos conhecimentos adquiridos nas aulas e seminários sobre o assunto. Sem esses serviços, a prescrição dos contraceptivos fica diluída nos ambulatórios de Ginecologia Geral.

A insegurança em relação ao aprendizado durante a graduação como um todo aparece quando a maioria dos médicos referiu ter frequentado um curso preparatório para a prova de residência e, também, pelo fato de a maioria dos médicos residentes recém-chegados à residência não se sentir segura para prescrever os ACH nas situações estudadas. Isso aponta dificuldades do curso de graduação em Medicina em conseguir conectar o conhecimento teórico e prático numa linha eficaz para garantir segurança à prática médica logo após a graduação e para as provas de residência. Assim, torna-se necessária a complementação do aprendizado com esse tipo de curso ${ }^{40,41}$.

Quando se compararam as taxas de acertos dos médicos encontradas no presente estudo com as dos graduandos de Medicina de uma faculdade de Medicina de Goiânia, em publicação de 2015, observou-se uma melhoria nas taxas. Quanto à contracepção de hipertensas graves, de hipertensas leves, de cefaleias com sinais de focalização, de antecedentes de tromboembolismo pessoal e tabagismo maior que 15 cigarros por dia, as taxas de acertos dos graduandos foram, respectivamente, de $9,7 \%, 3 \%, 13 \%, 12 \%$ e $11 \%$. Já entre os médicos residentes, no vigente estudo, essas taxas foram maiores, respectivamente, de $24,2 \%, 15,2 \%, 27,3 \%, 18,2 \%$ e $21,2 \%$, apresentando baixo índice de acertos, não havendo diferenças significativas entre os residentes do primeiro e últimos anos.

Como o fator que se interpôs entre a graduação e a residência foi o curso preparatório para residência médica, pode-se concluir que este tipo de curso pode estar concorrendo para a sedimentação teórica dos aspectos estudados. Um estudo feito entre estudantes de Medicina com o objetivo de conhecer a opinião deles sobre os cursos de residência médica, publicado em 2011, evidenciou que 91\% dos entrevistados reconheceram que o estudo teórico, com a participação nos cursos preparatórios, foi o fator mais importante para a aprovação nas provas de residência. Porém, quando o enfoque do estudo foi a formação profissional, 92,5\% afirmaram serem mais importantes as atividades práticas ${ }^{40}$.

Esses aspectos também podem explicar a maior porcentagem de acertos quando o método contraceptivo foi considerado correto, isoladamente, para cada situação estudada. Nessa condição, cada médico residente soube indicar um ou outro método como correto, e, juntos, os médicos conseguiram identificar todos os métodos corretos. Porém, quando as questões foram consideradas totalmente corretas apenas quando foram marcados todos os métodos corretos para cada situa- 
ção estudada, as taxas de acertos baixaram significativamente. Esses resultados também foram encontrados em estudo com os graduandos de Medicina ${ }^{35}$, mostrando as mesmas dificuldades em relação à organização do conhecimento, de forma integrada, necessária para acertar como um todo cada questão específica.

O baixo índice de acerto dos médicos residentes quando se consideraram todos os métodos corretos demonstra deficiência de conhecimento para oferecer opções terapêuticas às pacientes nas situações específicas abordadas neste estudo. Sabendo que variadas opções de tratamento são importantes para que haja melhor adesão ao adequar a mulher ao método, pode-se inferir que o desconhecimento de outras opções pode levar a baixa adesão ao método e a drásticas consequências, como gravidez indesejada.

Se a correta prescrição de determinado contraceptivo para cada situação específica é importante, deixar de prescrever um contraceptivo que poderia ser usado nessas situações é mais importante ainda. Deixar mulheres com situações de risco para gestação sem utilizar um método que elas poderiam empregar significa deixá-las correr o risco de uma gestação indesejada e não programada ${ }^{36}$.

Por causa do componente estrogênico, o uso de contraceptivos hormonais combinados está proscrito nas seguintes situações especiais: mulheres com HAS leve a grave, antecedentes pessoais de tromboembolismo arterial, cefaleia com sinais de focalização e tabagistas de mais de 15 cigarros/dia ${ }^{42,43}$. No entanto, nestes casos, não há contraindicações para o uso dos contraceptivos progestínicos isolados, uma vez que seus benefícios melhoram a qualidade de vida das mulheres e reduzem os danos associados a morbidade e mortalidade neonatais e as consequências negativas relacionadas às gestações indesejadas ${ }^{33}$.

Finalmente, após exaustivas pesquisas bibliográficas em sites de busca e nas bases de dados (Scielo, PubMed, Medline e Lilacs) sobre o assunto em questão, verificou-se que poucos estudos avaliam o conhecimento de profissionais de saúde sobre a contracepção hormonal, principalmente entre profissionais médicos. Estudos futuros sobre este aspecto serão importantes para elaborar medidas e estratégias que possam melhorar a prescrição de contraceptivos hormonais por parte do profissional da área de saúde.

\section{CONCLUSÃO}

Este estudo mostrou que os anos de residência médica não foram suficientes para melhorar significativamente os conhecimentos a respeito dos Critérios Médicos de Elegibilidade para Uso de Anticoncepcionais da OMS entre os grupos iniciais e finais do curso de residência, apesar de oferecerem maior segurança na prescrição. Contudo, em comparação com dados da literatura, os conhecimentos dos médicos residentes estudados foram maiores do que os encontrados entre alunos do internato de Medicina, apesar de apresentarem um importante déficit do conhecimento dos futuros ginecologistas e obstetras.

Ademais, este estudo evidenciou baixa taxa de acerto dos médicos em relação aos CMEUA da OMS nas situações específicas de cefaleias, hipertensão arterial, trombose venosa e arterial e embolia pulmonar, demonstrando que grande parte dos médicos estudados fica restrita apenas a uma opção terapêutica de contraceptivos hormonais, o que pode diminuir a adesão ao tratamento de cada paciente, levando a sérias consequências, como a gestação indesejada.

A falta de serviços de planejamento familiar eficazes nas instituições estudadas, os quais constituem a principal forma de colocar em prática e sedimentar os conhecimentos teóricos adquiridos na residência, pode estar associada aos resultados encontrados. Entretanto, há necessidade de mais estudos, com populações maiores de residentes, para concluir as orientações que o presente estudo apresentou.

\section{AGRADECIMENTOS}

Agradecemos à Diretoria dos Hospitais e aos médicos residentes de Ginecologia e Obstetrícia das instituições estudadas, pelo apoio, receptividade e colaboração na execução do trabalho.

\section{REFERÊNCIAS}

1. Faria VE. Políticas de governo e regulação da fecundidade: conseqüências não antecipadas e efeitos perversos. In: CIÊNCIAS sociais hoje. São Paulo. ANPOCS, 1989. [capturado 15 Abr. 2016]; 62-103.

2. Pedro JM. A trajetória da pílula anticoncepcional no Brasil (1960-1980). Biblioteca de História das Ciências e da Saúde. [capturado 15 Abr. 2016]. Disponível em: <http:/ / bases.bireme.br/cgibin / wxislind.exe/iah / online/?IsisScript=iah/iah. $x$ is\&src $=$ google $\&$ base $=$ LILAC S\&lang=p\&nextAction $=$ lnk\&exprSearch $=600558 \&$ indexSe $\operatorname{arch}=\mathrm{ID}>$

3. Flandrin JL. O sexo e o Ocidente. São Paulo: Brasiliense; 1988.

4. Pedro JM. A experiência com contraceptivos no Brasil: uma questão de geração. Rev. Bras. Hist. [online].2003. [capturado 15 Abr. 2016]; 239-260. Disponível em:<http:/ / www.scielo.br/scielo.php?script=sci_arttext\&pid=S01020 $1882003000100010 \& \operatorname{lng}=\mathrm{en} \& \mathrm{nrm}=\mathrm{iso}>$. 
5. Paz ECM, Ditterich RG. O conhecimento das mulheres sobre os métodos contraceptivos no planejamento familiar. Rev Gestão \& Saúde.[online]. 2009. [capturado 15 Abr. 2016]; 1-10. Disponível em: http:/ /www.herrero.com.br/ revista/Edicao\%201\%20Artigo\%204.pdf

6. Blumenthal PD, Edelman A. Hormonal contraception. Obstet Gynecol. 2008. Sep;112(3):670-84. doi: 10.1097/ AOG.0b013e31818425b7. Review. PubMed PMID: 18757668. [capturado 15 Abr. 2016]. 670-684. Disponível em: <http:/ / www.ncbi.nlm.nih.gov/pubmed/18757668>

7. Weaver MA, Raymond EG, Baecher L. Attitude and Behavior Effects in a Randomized Trial of Increased Access to Emergency Contraception. Obstetrics and Gynecology 2009; 113(1) [capturado 15 Abr. 2016]; 107-116. Disponívelem: <http:/ / doi.org/10.1097/ AOG.0b013e318190c0fe>

8. Brito MB, Nobre F, Vieira CS. Contracepção hormonal e sistema cardiovascular. Arq. Bras. Cardiol. [online]. 2011. [capturado 15 Abr. 2016]; 81-89. Disponível em:<http:/ / www.scielo.br/scielo.php?script=sci_arttext\&pid=S00667 82X2011000400021\&lng=en\&nrm=iso $>$.

9. Flores MBR. Entre a casa e a rua... memória feminina das festas açorianas no sul do Brasil. Cadernos Pagu. [online].1995. [capturado 15 Abr. 2016]; 117-142. Disponível em: <file:// /C:/Users/Ricardo/Downloads/1765-21461-SM.pdf $>$.

10. Bock G,ThaneP. La mujer en los estados de bienestar europeos, 1880-1950. Maternidad y políticasde género. Cátedra, Madri.[capturado 15 Abr. 2016].

11. Koven S, Michel S. Womanly duties: maternalist politics and the origins of welfarestates in France, Germany, Great Britain and the United States, 1880-1920. American Historical Review. [online]. 1990. [capturado 16 Abr. 2016]; 10761108. Disponívelem:< http://hrcolumbia.org/gender/papers/michel_maternalism_and_beyond.pdf $>$

12. Cohen $M$, Hanagan $M$. The politics of gender and the making of the welfare state, 1900-1940: A Comparative Perspective. Journal of Social History. [online]. 244 (Spring 1991) [capturado 16 Abr. 2016]; 469-84.Disponívelem: <http://jsh. oxfordjournals.org/content/24/3/469.extract>

13. Mclaren A. Historia de los anticonceptivos. Madrid: Minerva, 1993.

14. Pletzer BA,Kerschbaum HH. A short history of hormonal contraception. Front Neurosci. [online]. 2014.[capturado 16 Abr. 2016]. Disponível em: <http:/ / www.ncbi.nlm.nih. gov/pmc/articles/PMC4139599>.

15. Lidegaard O,Lokkegaard E,Svendsen AL,Agger C. Hormonal contraception and risk of venous thromboembolism: national follow-up study. BMJ. [online]. 2009. [cap- turado 14 Abr. 2016]. Disponível em: <http://www.ncbi. nlm.nih.gov/pubmed/19679613>.

16. WHO. Effect of different progestagens in low oestrogen oral contraceptives on venous thromboembolic disease. World Health Organization Collaborative Study of Cardiovascular Disease and Steroid Hormone Contraception. Lancet. [online]. 1995. [capturado 14 Abr. 2016].1582-1588. Disponível em: <http://www.ncbi.nlm.nih.gov/pubmed/7500749>.

17. Schindler AE, Campagnoli C, Druckmannc R, Huber J, Pasqualini JR, Schweppef KW et al. Classification and pharmacology of progestins. Maturitas. [online]. 2003. [capturado 14 Abr. 2016];171-80. Disponível em: <http:/ / www.ncbi.nlm.nih.gov/pubmed/14670641>.

18. Dericks-Tan JS, Schneider K, Taubert HD. The mechanism of action of a new low-dosed combined oral contraceptive. Arch Gynecol.[online]. 1980. [capturado 14 Abr. 2016]; 107-114. Disponível em: <http://www.ncbi.nlm.nih.gov/ pubmed/6768343>.

19. Moutos D; Smith S;Zacur H. The effect of monophasic combinations of ethinyl estradiol and norethindrone on gonadotropins, globulin: a randomized trial. Contraception. [online]. 1995.[capturado 15 Abr. 2016];105-109. Disponível em: <http:/ / www.ncbi.nlm.nih.gov/pubmed/8536446>.

20. Ware RS. New progestagens for contraceptive use. Hum Reprod Update. [online]. 2006. [capturado 15 Abr. 2016];169-78. Disponível em: <http://www.ncbi.nlm.nih. gov/pubmed/16291771>.

21. Wiegratz I, Kuhl H. Metabolic and clinical effects of progestogens. Eur J ContraceptReprod Health Care. [online]. 2006. [capturado 15 Abr. 2016]; 153-61.Disponívelem: <http://www.ncbi.nlm.nih.gov/pubmed/17056444>.

22. Vigo F, Lubianca JN, Corleta HE. Progestógenos: farmacologia e uso clínico. Femina.[online]. 2011. [capturado 15 Abr. 2016]. Disponível em: <http://files.bvs.br/ upload/S/0100-7254/2011/v39n3/a2498.pdf>.

23. Ware RSR. Pharmacological profile of progestins. Maturitas. [online]. 2008. [capturado 16 Abr. 2016];151-157. Disponível em: <http://www.ncbi.nlm.nih.gov/pubmed/19434887>.

24. Krattenmacher R. Drospirenone: pharmacology and pharmacokinetics of a unique progestogen. Contraception. [online]. 2000. [capturado 16 Abr. 2016];29-38. Disponível em:<http:/ / www.ncbi.nlm.nih.gov/pubmed/11024226>.

25. Wiegratz I,Kuhl H. Metabolic and clinical effects of progestogens. Eur J ContraceptReprod Health Care. [online]. 2006. [capturado 16 Abr. 2016]; 153-61.Disponívelem: <http://www.tandfonline.com/doi/abs/10.1080/ $1362518060077274>$. 
26. Macedo CSV. Efeito do implante de etonogestrel sobre o sistema hemostático de mulheres hígidas. Ribeirão Preto; 2006. Doutorado [Tese] - Faculdade de Medicina de Ribeirão Preto, Universidade de SãoPaulo.

27. Practice Committee of American Society for Reproductive Medicine. Hormonal contraception: recent advances and controversies. FertilSteril. [online]. 2008. [capturado 14 Abr. 2016];103-113. Disponível em: <http://www.reproductivefacts.org/uploadedFiles/ASRM_Content/News_ and_Publications/Practice_Guidelines/Educational_Bulletins/Hormonal_contraception_\%20recent(1).pdf $>$.

28. Poli MEH, Mello CR, Machado RB, Neto JSP , Spinola PG, Tomas G, Silveira MM, Formiga Filho JFN , Ferrari AEM, Giordano MV, Aldrighi JM, Giribela AHG, Araújo FF, Magalhães J, Bossemeyer RP. Manual de anticoncepção Febrasgo. FEMINA. [online]. 2009.[capturado 10 Mar. 2016]. Disponível em: <http://www.febrasgo.org.br/site/wp-content/uploads/2013/05/Femina-v37n9_Editorial.pdf>.

29. Magnusdóttir EM, Bjarnadóttir RI, Önundarson PT,Gudmundsdóttir BR,Geirsson RT, Magnusdóttir SD,Dieben TOM.The contraceptive vaginal ring (NuvaRing) and hemostasis: a comparative study. Contraception. [online]. 2004. [capturado 12 Mar. 2016]; 461-67. Disponível em: <http:/ / www.ncbi.nlm.nih.gov/pubmed/15157790>.

30. Jick H, Kaye JA,Scaramozza CV,Jick SS. Risk of venous thromboembolism among users of third generation oral contraceptives compared with users of oral contraceptives with levonorgestrel before and after 1995: cohort and case-control analysis.BMJ. [online]. 2000. [capturado 10 Mar. 2016];1190-1195. Disponível em: <http://www.ncbi.nlm. nih.gov/pubmed/11073511>.

31. Maia HJ,Casoy J. Non-contraceptive health benefits of oral contraceptives. Eur J ContraceptReprod Health Care. [online]. 2008.[capturado 13 Mar. 2016];17-24.Disponívelem: <http://www.ncbi.nlm.nih.gov/pmc/articles / PMC3693657>.

32. World Health Organization. US Medical eligibility criteria for contraceptive use. [online]. 2010. [capturado $10 \mathrm{Abr}$. 2016]. Disponível em: <http://www.cdc.gov/mmwr/ $\mathrm{pdf} / \mathrm{rr} / \mathrm{rr} 59 \mathrm{e} 0528 . \mathrm{pdf}>$.

33. Berrones S, Ángel M. Conocimiento, actitud y percepción de los médicos residentes de Ginecología y Obstetricia del ISSSTE acerca de la anticoncepción de emergencia. Rev de Especialidades Médico-Quirúrgicas. [online]. 2011. [capturado 13 Abr. 2016]. Disponível em: :<http:/ / www.redalyc. org/articulo.oa?id=47321154005>.

34. Alano GM, Costa LN, Miranda LR, Galato D. Conhecimento, consumo e acesso à contracepção de emergência entre mulheres universitárias no sul do Estado de Santa Catarina. Ciência \& Saúde Coletiva. [online]. 2012. [capturado 12 Mar. 2016]; 2397-2404.Disponível em: <http:/ / www.scielo.br/scielo.php?script=sci_arttext\&pid $=$ S1413-81232012000900020 $>$.

35. Giglio MRP, Andrade LC,Daher GM, Ribeiro MO, Albernaz MA. Contracepção hormonal segundo a ótica do estudante de medicina: Mais um desafio para o ensino médico brasileiro?. Rev. bras. educ. med. [online]. 2015. [capturado 12 Abr. 2016];502-506. Disponível em: <http:/ /dx.doi. org/10.1590/1981-52712015v39n4e02952014>.

36. Dehlendorf C, Levy K, Ruskin R, Steinauer J. Health care providers' knowledge about contraceptive evidence: A barrier to quality family planning care? Contraception. [online]. 2010. [capturado 13 Abr. 2016]; 292-298. Disponível em: <http://www.ncbi.nlm.nih.gov/pmc/articles / PMC2892417/pdf/nihms159184.pdf>.

37. Suzanne C, Smeltzer OC, Brenda G, Janice L. Use of hormonal contraception in women with coexisting medical conditions. Practice Bulletin No. 73: American College Of Obstetricians And Gynecologists. Obstetrics and Gynecology. [online]. 2006.[capturado 16 Abr. 2016]; 1453-1472.

38. Isaacs JN, Creinin MD. Miscommunication between healthcare providers and patients may result in unplanned pregnancies. Contraception. [online]. 2003. [capturado 16 Abr. 2016];373-376. Disponível em: <http:/ / www.sciencedirect.com/science/article/pii/S0010782403002270>.

39. Lourenção LG,Moscardini AC, Soler ZASG. Saúde e qualidade de vida de médicos residentes. Rev Assoc Med Bras. [online]. 2010. [capturado 13 Abr. 2016];81-91. Disponível em: <http:/ / www.scielo.br/pdf/ramb/v56n1/21.pdf>

40. Silva SM, Rosa VF, Brandão PRP, Oliveira AC, Oliveira PG, Sousa JB. Cursos preparatórios para a residência médica: visão dos estudantes de medicina. Rev do Colégio Brasileiro de Cirurgiões. [online]. 2011. [capturado 14 Abr. 2016]; 349-354. Disponível em: <http://www.scielo.br/scielo. php?script=sci_arttext\&pid=S0100-69912011000500012>.

41. Magalhães CEV. Concurso de seleção para residência médica entre aluno de graduação e especialista. RevAngiolCir Vasc.[online]. 2006. [capturado 14 Abr. 2016];80-82. Disponível em: <http://www.santacasabh.org.br/app/ webroot/files/uploads/Catalogo_WEB.pdf $>$.

42. Grossman D, White K, Hopkins K, Amastae J,Shedlin M, Potter JE. Contraindications to combined oral contraceptives among over-the-counter compared with prescription users.Obstet Gynecol. [online]. 2011. [capturado 10 Abr. 2016];558-65. Disponível em: <https://www.researchgate.net/publication/49857330_Contraindications_to_Com- 
bined_Oral_Contraceptives_Among_Over-the-Counter_ Compared_With_Prescription_Users>.

43. White K, Potter JE, Hopkins K, Fernández L, Amastae J, Grossman D. Contraindications to progestin-only oral contraceptive pills among reproductive-aged women. Contraception. [online]. 2012. [capturado 11 Abr. 2016];199-203. Disponível em: <http://www.ncbi.nlm.nih.gov/pubmed/22364816>.

\section{CONFLITO DE INTERESSES}

As autoras declaram não haver conflito de interesses na realização deste estudo.

\section{CONTRIBUIÇÃO DOS AUTORES}

Margareth Rocha Peixoto Giglio: contribuição científica e intelectual efetiva para o estudo; concepção e delineamento; interpretação dos dados; procedimentos técnicos; análise estatística; preparação do manuscrito; redação do manuscrito; revisão crítica; aprovação final. Gisele Pimenta Melo: contribuição científica e intelectual efetiva para o estudo; concepção e delineamento; interpretação dos dados; procedimentos técnicos; análise estatística; preparação do manuscrito; redação do manuscrito; revisão crítica; aprovação final. Vanessa Guerra Ferreira: contribuição científica e intelectual efetiva para o estudo; concepção e delineamento; interpretação dos dados; procedimentos técnicos; análise estatística; preparação do manuscrito; redação do manuscrito; revisão crítica; aprovação final. Marco Aurélio Albernaz: revisão bibliográfica; revisão crítica. Marilia Oliveira Ribeiro: coordenadora da coleta de dados; revisão crítica.

\section{ENDEREÇO PARA CORRESPONDÊNCIA}

Margareth Rocha Peixoto Giglio

Departamento de Medicina da PUC Goiás

Av. Universitária, 1069 - Setor Universitário

Setor Leste Universitário - Goiânia

CEP 74605-010 - GO

E-mail: margarethgiglio@uol.com.br; vanessaguerraferreira@ gmail.com; gijapimenta@gmail.com 